\title{
Lymphedema techniques to manage edema after SCl: a retrospective analysis
}

\author{
Rebecca Hammad $^{1} \cdot$ Catherine Furbish $^{1} \cdot$ W. Mark Sweatman ${ }^{1} \cdot$ Edelle C. Field-Fote $^{1,2,3}$
}

Received: 4 January 2018 / Revised: 22 March 2018 / Accepted: 31 March 2018 / Published online: 12 June 2018

(c) International Spinal Cord Society 2018

\begin{abstract}
Study design Retrospective analysis of treatment data for a cohort of clients with spinal cord injury (SCI) who received therapy for management of edema.

Objective To evaluate the safety, feasibility, and benefit of a modified lymphedema treatment approach for treatment of chronic lower extremity edema in persons with SCI.

Setting A specialty rehabilitation hospital in Atlanta, GA, USA.

Methods Certified lymphedema therapists with experience in SCI rehabilitation modified standard complete decongestive therapy (CDT) techniques to accommodate sensory and motor impairments and ensure skin safety. Therapists applied the modified CDT (mCDT) approach as part of treatment in 59 adults with SCI and lower extremity edema. Limb volume was measured using standardized volumetric measurement, pitting was scored using a standardized scale (range 0-4), and edema characteristics were determined to be present or absent. Outcomes of the mCDT intervention were analyzed for 105 lower extremities.

Results Outcomes indicated that mCDT was associated with significant reduction in limb volumes, with a mean decrease of $11 \pm 7.6 \%$. Significant decreases were also observed in pitting edema and edema-specific characteristics, mean pitting scale score was reduced from a 3/4 to a 1/4. Minor adverse events were identified in a small number of patients.

Conclusions We found the mCDT approach to be safe and well-tolerated by the patients with SCI. The intervention was associated with decreased edema, and was feasible for use in a clinical setting. We recommend considering this mCDT approach for management of edema in individuals with SCI, while remaining vigilant about skin inspection.
\end{abstract}

\section{Introduction}

Each year, between 250,000 and 500,000 people in the world sustain spinal cord injuries (SCI) [1]. There are $\sim 282,000$ persons living with chronic SCI in the US alone [2]. Thirty-nine percent of individuals with chronic SCI

Electronic supplementary material The online version of this article (https://doi.org/10.1038/s41393-018-0141-8) contains supplementary material, which is available to authorized users.

Rebecca Hammad

Rebecca.Hammad@Shepherd.org

1 Crawford Research Institute, Shepherd Center, Atlanta, Georgia

2 Department of Rehabilitation Medicine, School of Medicine, Emory University, Atlanta, Georgia

3 Program in Applied Physiology, School of Biological Sciences, Georgia Institute of Technology, Atlanta, Georgia who use a wheelchair report edema as a secondary health condition, and this percentage increases with time since injury [3]. Impaired mobility and wheelchair use are associated with prolonged time spent in a seated position; as a result, these individuals are at risk for gravity-dependent edema in their lower extremities [4]. Persons with SCI are also at an increased risk for venous thromboembolism (VTE) [5], which further increases their risk of edema.

Edema adds to the burden of disability, impacting the ability to complete daily routines in numerous ways. The increase in weight of affected limbs makes transfers and other mobility tasks more difficult. Edema results in decreased joint mobility and range of motion further impairing mobility. Forty-five percent of persons with SCI indicate that edema causes them difficulty with function [6]. Beyond mobility, edema increases the potential for compromised skin integrity and infection.

Typical treatments for dependent edema include elevation, retrograde massage, compression, functional electrical 
stimulation, kinesiology tape, pneumatic pumps, and diuretics [7-10]. An extensive literature search revealed few published studies related to the management of edema in persons with SCI. Treatments noted in the SCI literature include elevation and gradient compression stockings [11, 12]. To the best of our knowledge there are no prior studies related to persistent lower extremity edema in individuals with SCI.

Complete decongestive therapy (CDT) is the standard therapeutic intervention for the management of lymphedema. Lymphedema is a condition in which there is an abnormal accumulation of protein-rich fluid in the interstitial spaces caused by impairment of the lymphatic system, leading to swelling and trophic changes of the skin. This condition can be hereditary, but is also common following lymph node removal or radiation therapy. CDT is comprised of an intensive phase and a maintenance phase. The intensive phase involves four components: skin care, daily range of motion and breathing exercises, manual lymphatic drainage (MLD), and compression wrapping with multi-layered short-stretch bandaging. In the maintenance phase, compression garments replace bandaging [13].

CDT is not commonly used for management of edema in individuals with neurological impairment. According to Suehiro [4], edema in persons with limited mobility is most commonly caused by venous stasis and is often accompanied by the trophic skin changes that are typical in chronic venous insufficiency. Since the swelling and trophic skin changes observed in these individuals is comparable to that in conditions for which CDT is indicated, the application of this approach in persons with edema due to limited mobility seems appropriate.

Our objective was to evaluate the safety, feasibility, and benefit of a modified lymphedema treatment approach for treatment of dependent edema and VTE-related edema in persons with SCI. We performed a retrospective analysis of 59 patients with SCI and lower extremity edema who were treated with an mCDT approach. We evaluated limb volume and edema-related characteristics prior to and following the CDT intervention.

\section{Methods}

A certified lymphedema therapist (CLT) who was also a licensed occupational therapist with experience in SCI rehabilitation originated an approach wherein standard CDT procedures were modified for use in individuals with SCI. Based on favorable early outcomes, additional occupational, and physical therapists pursued training to become certified as lymphedema therapists. The team worked together to further refine the mCDT approach. With increasing recognition of the value to patients, the hospital instituted a
Lymphedema Program and physicians referred patients for the intervention. Patients were not considered for this intervention if they had any condition that would contraindicate CDT, including: congestive heart failure, acute renal failure, untreated deep vein thrombosis, active cancer, swelling of unknown etiology, or acute infection.

Between April 2015 and February 2017, the first 2 years of the edema program, 2100 SCI patients received physical and/or occupational therapy in inpatient, post-acute or outpatient programs. Of these, $97(5 \%)$ were referred for mCDT for either upper or lower extremity edema. Consecutive patients were treated if a physician had ordered CDT for edema management after more conventional intervention had failed. A total of 59 (3\%) patients received the lower extremity intervention during the period covered by this analysis. The institutional review board at Shepherd Center approved this analysis of a pre-existing de-identified data set.

\section{Intervention}

Patients who met the inclusion criteria underwent a comprehensive CDT program of 3-5 sessions per week for 1-3 weeks depending on edema severity and response to treatment. All CDT treatments were rendered by a certified CLT who was also a licensed occupational or physical therapist.

The intensive phase of the CDT program included four components:

1. Manual lymphatic drainage. MLD was initiated using diaphragmatic breathing with overpressure. Subclavianinternal jugular junctions and inguinal node beds were stimulated. Specialized massage techniques were then used to assist the lymphatic system to mobilize fluid. If necessary, manual techniques to break down fibrotic areas were incorporated. Each session included $~ 10 \mathrm{~min}$ of MLD.

2. Compression therapy. A multi-layered bandaging technique was used to reduce limb volume and reverse trophic changes. The objective was to achieve the proper compression gradient through the systematic use of padding, shaping, and layering of short-stretch bandages (Rosidal K, Lohmann \& Rauscher, Topeka, KS). This method supports lymphatic function and helps prevent reaccumulation of fluid; the bandages were not intended to squeeze fluid out of the congested area. Bandages are worn $23 \mathrm{~h}$ per day. In our patients, compression was applied with modifications as follows (Fig. 1):

(a) Cotton padding placed across top of foot, bend of ankle and along the tibia.

(b) Increased layers of rolled cotton padding with limited use of foam padding.

(c) Toe wraps over foot padding instead of directly on the skin. 
Fig. 1 Compression bandaging -modifications to standard padding and bandaging techniques

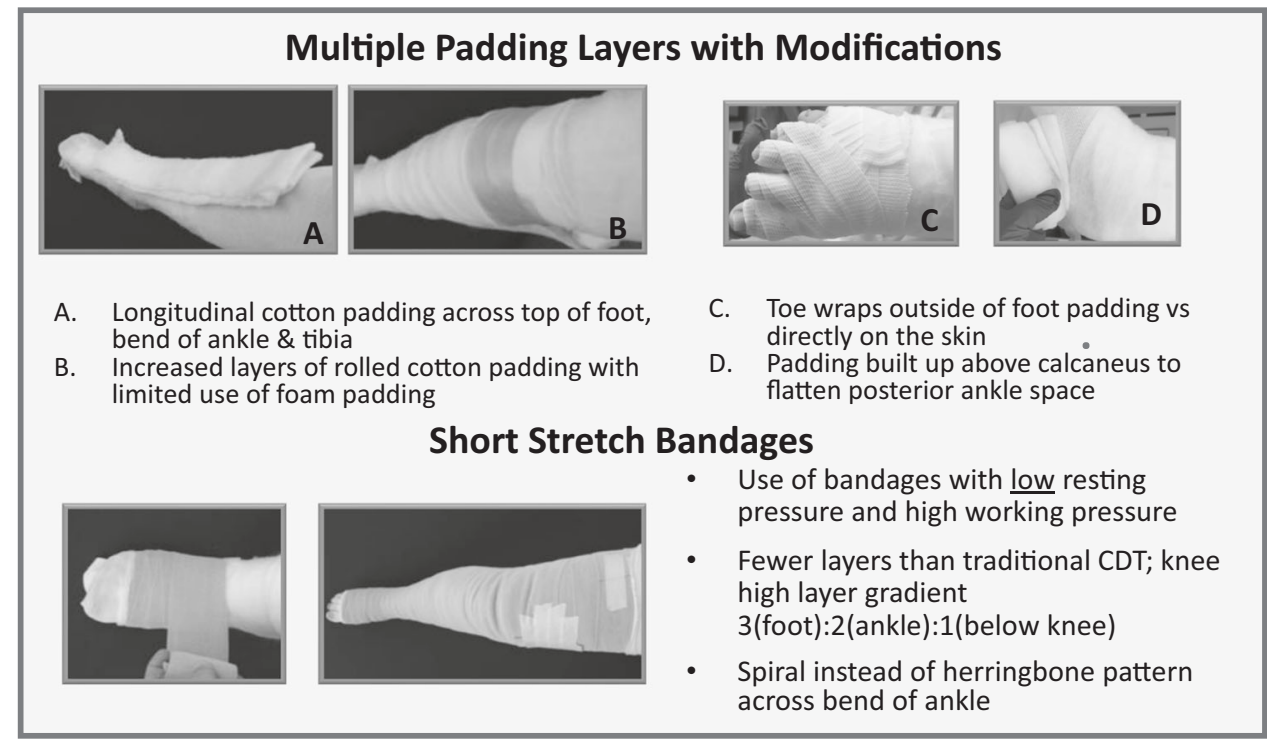

(d) Padding built up above calcaneus to flatten posterior ankle space.

(e) Fewer layers of short-stretch bandages than traditional CDT; knee-high layer gradient-3(foot): 2(ankle): 1(below knee).

(f) Spiral instead of herringbone pattern across bend of ankle to decrease layers and, thereby, decrease pressure.

3. Remedial exercises. In CDT, the terminology "remedial exercises" refers to basic exercises intended to promote lymphatic motility. The technique began with 10-15 deep, diaphragmatic breaths against manual resistance. Diaphragmatic breathing exercises are used to stimulate deep lymphatic structures, such as the cisterna chyli, the abdominal part of the thoracic duct, lumbar trunks and lumbar lymph nodes, pelvic lymph nodes, and certain organ systems. Stimulation of these deep lymphatic structures, particularly the thoracic duct (the largest lymph vessel in the body), accelerates the transport of lymph fluid toward the venous angles, through which the lymph fluid is returned into the blood circulatory system. Lymph fluid from the lower extremities passes through these deep lymphatic structures and an increased flow of lymph results in improved lymphatic drainage from the lower extremities. During exercise, deep breathing continued during 15-20 min of simple, sequential movements of the lower extremity joints, moving from proximal to distal to proximal. For patients with volitional control, active movements were utilized to incorporate muscle pump activity. Activeassisted or passive movements were substituted as necessary. Exercises were performed with bandages on to further assist with lymphatic motility.

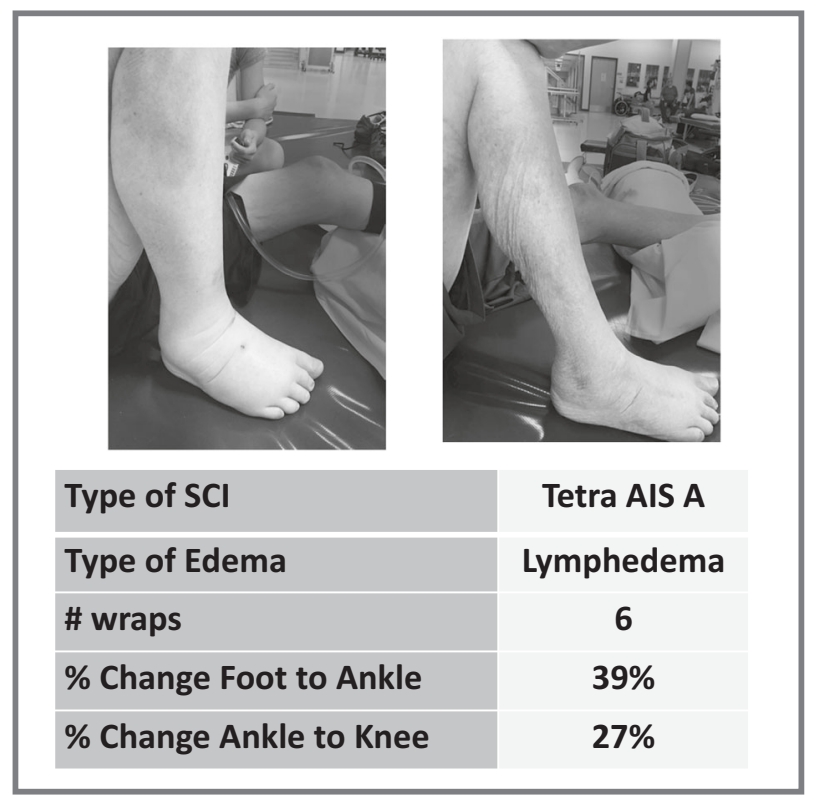

Fig. 2 Patient with tetraplegia and lymphedema

4. Instructions for skin and nail care, as needed. The main principles for skin care included in the instruction were to prevent dry or cracking skin; use lotions and soaps free of perfume, fragrance, or alcohol; ensure meticulous nail care; observe skin for signs/symptoms of infection; and treat infections and wounds promptly.

The intensive phase of CDT treatment ended when the CLT determined that there was no further volume decrease with bandaging and when edema characteristics had diminished (Figs. 2 and 3). Following the intensive phase of the CDT program, patients entered the maintenance phase. They were fitted with either standard or custom gradient 
Fig. 3 Patient with paraplegia and dependent/VTE-related edema

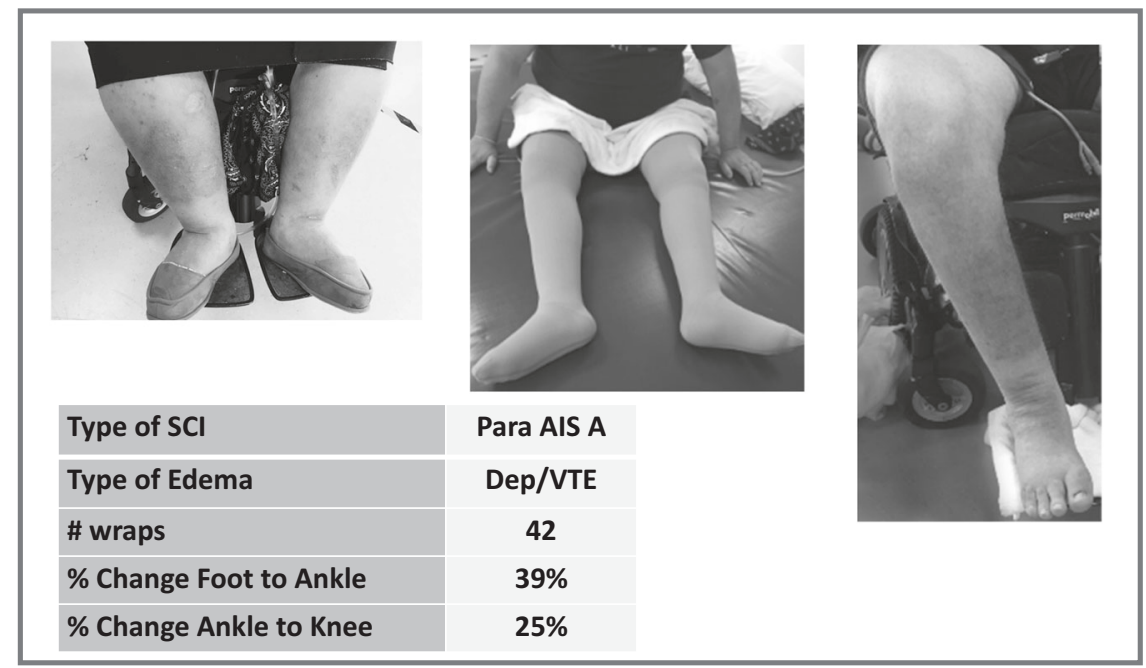

\begin{tabular}{lll}
\hline Type of SCI & Tetraplegia $(n=31) 52.5 \%$ & Paraplegia $(n=28) 47.5 \%$ \\
Motor completeness & Motor complete (AIS A +B) $(n=35)$ & Motor incomplete (AIS C + D) $(n=24)$ \\
& $59.3 \%$ & $40.7 \%$ \\
Edema & Bilateral edema $(n=46) 78 \%$ & Unilateral edema $(n=13) 22 \%$ \\
\hline
\end{tabular}

$n$ number of patients

compression stockings and were instructed in appropriate home maintenance programs. The maintenance phase of CDT continues indefinitely in persons with chronic lymphedema. In the SCI population, the maintenance phase will also continue indefinitely due to the chronicity of SCI and the associated immobility.

\section{Assessment}

Assessments were conducted at the initial evaluation (baseline) and upon removal of bandages at the last visit of the intensive program (final). Assessments included:

1. Fluid volume: Circumferential measurements were taken at the great toe, across the metatarsals, at mid-foot arch, at the malleoli, and every $8 \mathrm{~cm}$ up the leg, assuring a measurement at the middle of the patella and at the top of the leg, just below the gluteal fold. Volume was calculated from circumference and length measurements using truncated cone formula. The reliability and specificity of the calculated volume has previously been established [14].

2. Pitting scale: Pitting is a phenomenon wherein finger impressions remain on the skin after it has been pressed. Scores range from 0 to 4 , wherein 0 indicates no pitting edema. A score of 1 suggests mild pitting, such that a $2 \mathrm{~mm}$ depression disappears rapidly. A score of 2 indicates moderate pitting wherein a $4 \mathrm{~mm}$ depression disappears in $10-15 \mathrm{~s}$. A score of 3 suggests moderately severe pitting, such that a $6 \mathrm{~mm}$ depression may last more than $1 \mathrm{~min}$. A score of 4 indicates severe pitting edema such that an $8 \mathrm{~mm}$ depression can last more than $2 \mathrm{~min}$.

3. Observational data: The following characteristics were noted as present or absent:

3a. General Edema Characteristics: redness, discoloration, fibrosis, lesions, scarring, taut or shiny skin, and weeping

3b. Lower Extremity-Specific Edema Characteristics: loss-of-normal skin wrinkles, oblivion of extensor tendons, presence of dorsal foot hump, lack of ankle contour, and positive Stemmer's sign (a thickened fold of skin at the based of the second digit that can be pinched and lifted, indicating increased density/fibrosis).

4. Type of Edema: Based on patient medical history and observational data, CLTs classified the type of edemadependent, VTE-related, lymphedema or a combination. When the edema was due to the dependent position of the lower extremity in the absence of known VTE or previously diagnosed lymphedema the edema was classified as "dependent." When a VTE was identified as acute or chronic in the extremity the edema was classified as "VTErelated." When lymphedema was previously diagnosed prior to the SCI the edema was classified as "lymphedema." In other cases of "lymphedema" the edema was chronic and had developed into lymphedema demonstrating a positive Stemmer's sign, trophic changes, and pitting.

In addition to documenting the outcome measures of interest, any adverse events were recorded on the assessment forms. 
Table 2 Fluid volume outcomes -volumes in cc, change values, Cohen's $d$ and $p$-values

\begin{tabular}{|c|c|c|c|c|c|c|}
\hline Segment & $\begin{array}{l}\text { Initial volume } \\
\text { (cc) mean } \\
\text { (SD) }\end{array}$ & $\begin{array}{l}\text { Final volume } \\
\text { (cc) mean } \\
\text { (SD) }\end{array}$ & $\begin{array}{l}\text { Volume } \\
\text { change (cc) } \\
\text { mean (SD) }\end{array}$ & $\begin{array}{l}\text { Volume } \\
\text { change } \% \\
\text { mean (SD) }\end{array}$ & Cohen's $d^{\mathrm{b}}$ & $p$-value \\
\hline Foot to ankle & $500(173)$ & 429 (124) & $71(67)$ & $13 \%(8.2 \%)$ & 0.47 & $<0.001$ \\
\hline Ankle to knee & $4182(1426)$ & 3689 (1046) & $493(661)$ & $11 \%(7.7 \%)$ & 0.39 & $<0.001$ \\
\hline Foot to knee & $4682(1556)$ & 4118 (1123) & $564(710)$ & $11 \%(7.5 \%)$ & 0.42 & $<0.001$ \\
\hline Knee to groin & $6381(2301)$ & $5685(1701)$ & 696 (1042) & $8 \%(10.7 \%)$ & 0.34 & $<0.001$ \\
\hline $\begin{array}{l}\text { Foot to groin } \\
\text { (full lower } \\
\text { extremity) }\end{array}$ & 6809 (4146) & 6013 (3353) & 796 (1199) & $11 \%(7.6 \%)$ & 0.21 & $<0.001$ \\
\hline
\end{tabular}

${ }^{a}$ Percent change from initial volume to final volume

${ }^{\mathrm{b}}$ Effect size $($ small $=0.2$, medium $=0.5$, large $=0.8)$
Table 3 Observational data-general characteristics - changes from baseline to final assessment with Cohen's $d$ and $p$-values

\begin{tabular}{llllll}
\hline Characteristic & Initial & Final & Change $^{\mathrm{a}}$ & $p$-value & Cohen's $d^{\mathrm{b}}$ \\
\hline Redness & $14 \%$ & $1 \%$ & $13 \%$ & $<0.001$ & 0.50 \\
Discoloration & $29 \%$ & $6 \%$ & $23 \%$ & $<0.001$ & 0.64 \\
Fibrosis & $22 \%$ & $7 \%$ & $15 \%$ & $<0.001$ & 0.44 \\
Lesion Present & $13 \%$ & $7 \%$ & $6 \%$ & 0.008 & 0.20 \\
Scarring & $5 \%$ & $2 \%$ & $3 \%$ & 0.083 & 0.17 \\
Taut or shiny skin & $73 \%$ & $11 \%$ & $62 \%$ & $<0.001$ & 1.60 \\
Weeping & $2 \%$ & $0 \%$ & $2 \%$ & 0.158 & 0.21 \\
\hline
\end{tabular}

${ }^{\mathrm{a}}$ Change represented as a percent of subjects with an elimination of characteristic

${ }^{\mathrm{b}}$ Effect size $($ small $=0.2$, medium $=0.5$, large $=0.8)$

Statistical analyses via matched pairs $t$-test, significance accepted as $p<0.01$

$\%$ indicates $\%$ of patients in which the characteristic was present

\section{Statistical analyses}

Descriptive statistics were calculated for patient characteristics (Table 1), edema diagnosis, and number of wraps completed. Match pairs $t$-tests were employed to determine significant differences between initial and final lower extremity volume (Table 2), as well as percentage of patients who exhibited each general (Table 3) and lower extremity-specific characteristic (Table 4) of edema. Significant differences in pitting edema from initial to final wrap were determined by Wilcoxon signed-rank test due to the ordinal nature of the variable. Changes were considered significant at $p<0.05$. Change in percentage of patients with each characteristic was calculated, along with effect size (Cohen's $d$ ).

\section{Results}

Retrospective analysis included 59 patients. Patient characteristics are listed in Table 1. Thirty-one (53\%) patients
Table 4 Observational data-lower extremity-specific characteristics -changes from baseline to final assessment with Cohen's $d$ and $p$-values

\begin{tabular}{|c|c|c|c|c|c|}
\hline Characteristic & Initial & Final & Change $^{\mathrm{a}}$ & $p$-value & Cohen's $d^{\mathrm{b}}$ \\
\hline $\begin{array}{l}\text { Loss-of-normal skin } \\
\text { wrinkles }\end{array}$ & $73 \%$ & $8 \%$ & $65 \%$ & $<0.001$ & 1.77 \\
\hline $\begin{array}{l}\text { Oblivion of extensor } \\
\text { tendons }\end{array}$ & $53 \%$ & $12 \%$ & $41 \%$ & $<0.001$ & 0.97 \\
\hline $\begin{array}{l}\text { Presence of dorsal } \\
\text { hump_foot }\end{array}$ & $42 \%$ & $4 \%$ & $38 \%$ & $<0.001$ & 1.01 \\
\hline Lack of ankle contour & $92 \%$ & $20 \%$ & $72 \%$ & $<0.001$ & 2.11 \\
\hline $\begin{array}{l}\text { Positive Stemmer's } \\
\text { sign }\end{array}$ & $15 \%$ & $6 \%$ & $9 \%$ & 0.001 & 0.3 \\
\hline
\end{tabular}

${ }^{a}$ Change represented as a percent of subjects with an elimination of characteristic

${ }^{\mathrm{b}}$ Effect size $($ small $=0.2$, medium $=0.5$, large $=0.8)$

Statistical analyses via matched pairs $t$-test, significance accepted as $p<0.01$

$\%$ indicates $\%$ of patients in which the characteristic was present

had tetraplegia and 28 (48\%) patients had paraplegia. Thirtyfive patients (nearly 60\%) had motor-complete injuries. Forty-six patients $(78 \%)$ had bilateral lower extremity edema. Of the patients with motor-complete injuries, 30 (86\%) experienced bilateral edema for which both lower extremities were treated and included in the analysis. Our analysis included a total of 105 lower extremities.

Patients presented with different types of edema, including: dependent edema $(n=72)$, dependent edema + VTE $(n=17)$, VTE edema $(n=8)$, lymphedema $(n=6)$, and lymphedema + VTE $(n=2)$. Some patients had a different type of edema in each lower extremity. The mean number of intensive phase treatments with bandaging was $6.7 \pm 7.9$.

\section{Limb volume}

Following the intensive phase of CDT, full lower extremity volumes (foot to groin) decreased a mean of $6013 \mathrm{ml}$ 
compared to baseline, representing an $11 \pm 7.6 \%$ reduction in volume. The largest percentage change in a lower extremity segment was observed in the foot- to- ankle segment. The mean change in this segment was $500 \mathrm{ml}$ or 13\%. All changes during the intensive phase were significant (Table 2).

\section{Pitting scale}

At baseline, the median pitting scale score was a 3, indicating moderately severe pitting edema such that a $6 \mathrm{~mm}$ impression may last more than $1 \mathrm{~min}$. The post-CDT median score was a 1 , indicating mild pitting edema such that a $2 \mathrm{~mm}$ impression disappears rapidly. (Cohen's $d=1.67$ )

\section{Observational data-general characteristics}

The presence of 5/7 general edema characteristics, including redness, discoloration, fibrosis, lesions, and tautness of skin, decreased significantly from initial to final assessments. Scarring and weeping were present in low percentages of patients at initial assessment; therefore, the change in the percentage of patients with these characteristics was not significant. (Table 3)

Lower extremity-specific characteristics. The percentage of patients exhibiting each of the five lower extremityspecific edema characteristics, loss of normal skin wrinkles, oblivion of extensor tendons, presence of dorsal foot hump, lack of ankle contours, and presence of a positive Stemmer's sign, decreased significantly. (Table 4)

\section{Adverse events}

No adverse events were observed during the intensive phase of CDT. However, several minor adverse events were observed during the maintenance phase. There were four cases of mild redness (stage I pressure injuries) over the anterior ankle joint line noted with the first wearing of compression garments. In 3 cases, modifications were made in fabric, compression level or size of the garment for a more appropriate fit. In the fourth case, a night garment (Biacare) was substituted for a standard daily compression garment. There was one case of an allergic reaction to the silicone band of a garment. In this case, the garment was replaced with a custom silicone-free garment.

\section{Discussion}

Our data show that the mCDT approach was associated with significant decreases in limb volumes and pitting edema, and in the percentage edema characteristics in the lower extremities of individuals with spinal cord injuries. Lower extremity volumes decreased a mean of $11 \%$. The median pitting scale score was reduced from a 3 to a 1 on a $0-4$ scale.

It has been suggested that for rehabilitation interventions, an effect size of $\geq 0.4$ is meaningful [15]. Outcomes related to fluid volume, observational data, and pitting quality all indicated improvement, meeting or exceeding $d=0.4$. Modified CDT was of value in treating lower extremity edema in patients with SCI.

In this cohort of patients, $85 \%$ had a dependent edema component, $26 \%$ had a VTE component and only $7.6 \%$ had a true lymphedema component. It can be assumed that individuals with only dependent and/or VTE-related edema had healthy, fully functional lymphatic systems. None of the patients with lymphedema components had been diagnosed with primary lymphedema prior to their SCI and none had family history of lymphedema. For this reason, it seems likely that their lymphedema was related to high-output lymphatic transport failure [16], also known as "lymphatic fatigue", caused by longstanding lower extremity edema or chronic VTE.

To our knowledge this is the first report of the use of CDT in individuals with a neurological condition. It is difficult to compare the results of the present study with those in the lymphedema literature for several reasons. The standard measure of lymphedema severity is percent of excess volume (PEV), which is calculated by comparing the baseline volume of the edematous limb $\left(V_{\mathrm{LB}}\right)$ to the baseline volume of the unaffected "healthy" limb $\left(V_{\mathrm{HB}}\right)$ of the same person: $\mathrm{PEV}=\left(\left(V_{\mathrm{LB}}-V_{\mathrm{HB}}\right) / V_{\mathrm{HB}}\right) \times 100 \%$. The outcome measure is percent reduction of excess volume (PREV), which is calculated using the equation: $\mathrm{PREV}=\left(\left(V_{\mathrm{LF}}-V_{\mathrm{LB}}\right) / \mathrm{PEV}\right) \times 100 \%$, (where ' $F$ ' is the final volume) [17]. In our study, most patients had bilateral lower extremity edema with no healthy limb for comparison. Our outcome measure was percent reduction in volume (PRV), which compared baseline limb volume $\left(V_{\mathrm{LB}}\right)$ to final, post-CDT, volume of the same limb $\left(V_{\mathrm{LF}}\right)$. $\mathrm{PRV}=\left(\left(V_{\mathrm{LB}}-V_{\mathrm{LF}}\right) / V_{\mathrm{LB}}\right) \times 100 \%$.

The International Society of Lymphology [16] defines lymphedema severity as minimal $(<20 \%$ PEV), moderate (20-40\% PEV) or severe ( $>40 \%$ PEV). In a study of the efficacy of CDT in lower extremity lymphedema [17], the average baseline PEV was of the moderate severity at $32.9 \%$. The same study found the average PREV to be $-55.1 \%$. This means that excess volume was reduced by $55 \%$. This is roughly comparable to an $18 \%$ reduction of limb volume. Our result of $11 \%$ reduction of limb volume is reasonably close to this volume considering that we used different calculation methods and that we used a modified approach in a different patient population.

Our modified intensive CDT sessions differed from standard CDT sessions in many ways. We utilized a 
pragmatic approach to treatments in a standard therapy gym. Due to scheduling constraints, patients were allotted only $1 \mathrm{~h}$ for each treatment. The mobility impairments of our patients increased the setup time required for patient positioning. For patient privacy, shorts were worn, thereby limiting access to some lymphatic pathways. A recent review of breast cancer-related lymphedema treatment [18] showed mixed results as to whether MLD offered any benefit when added to treatment with compression bandaging alone. For this reason, and due to time constraints, the MLD component of each treatment was limited to $\sim 10 \mathrm{~min}$ duration.

Other common approaches to edema in the SCI population are mentioned in the literature. After traumatic SCI, patients typically require spinal stabilization surgery. As is common with post-operative recovery, thromboprophylactic stockings (TPS), also known as thromboembolic deterrent (TED) hose, are utilized in this population to prevent VTE. TPS with a pressure range between 15 and $18 \mathrm{mmHg}$ should be differentiated from gradient compression stockings or garments that are specifically designed for patients with venous or lymphatic pathology. TPS are indicated during bedrest only. They are designed to decrease the venous diameter in the supine position, thereby increasing venous blood flow and avoiding blood flow stagnation [19]. The CLOTS1 trial [20] in stroke patients noted that $5.1 \%$ of neurologically impaired clients using thigh-high TPS developed skin compromise.

TPS are not designed to prevent dependent edema. For this reason, elastic bandages (e.g., ACE wraps or Coban) are often wrapped over the TPS to prevent edema or to reduce orthostatic hypotension in individuals with SCI. Elastic bandages are designed to stretch to $140 \%$ of their original length. This "long-stretch" characteristic causes this type of bandage to have constant high resting pressure. This pressure restricts superficial venous and lymphatic vessels. This can cause a tourniquet effect and can increase the risk for skin compromise in individuals with sensory impairment.

CDT bandaging utilizes short-stretch bandages (e.g., Comprilan or Rosidal K). These bandages stretch only to $60 \%$ of their length. Using bandages that have a low resting pressure reduces the risk for pressure injuries. Skin is also protected by using padding under bandaging and by utilizing gradient compression garments, in the maintenance phase, to eliminate bunching in contours or joint creases, thereby reducing the risk of tourniquet effect. In some cases, the more robust fabrics of custom, flat-knit garments are necessary to prevent bunching. In other cases, adjustments need to be made to wheelchairs to reduce flexion at the knee and/or ankle joints.

A small study in older individuals [21] found appropriate blood perfusion in the great toe before, during and after the use of short-stretch bandages (e.g., Comprilan) for lower extremity edema management. During CDT bandaging, the patient's toes are exposed, allowing one to check capillary refill and temperature to ensure circulation is not compromised. This is especially important to consider in the sensory impaired population.

Regular skin monitoring is essential for safely using CDT in persons with SCI. While there were no adverse events during the intensive phase, there were minor adverse events early in the maintenance phase during the transition to compression garments. Compression bandages are infinitely adjustable, while garments are not adjustable. Areas of redness and skin irritation are common during initial garment use even in fully sensate individuals with lymphedema. Another SCI-specific concern with CDT is autonomic dysreflexia caused by inability to drain the bladder. During a single MLD session (30-45 min) as much as $200-500 \mathrm{ml}$ fluid can be mobilized [13]. The lymphatic system will carry this extra fluid to the circulatory system and it will eventually be deposited in the bladder. It is important that individuals with SCI who have neurogenic bladders ensure that their bladders are drained before and after each CDT session.

\section{Limitations and recommendations for future study}

This study included individuals who received mCDT as part of their rehabilitation care, in some cases the individuals were admitted as in-patients specifically for the management of edema. For this reason, no attempt was made to compare the mCDT intervention to other approaches. However, all were referred to the mCDT treatment because other approaches to manage edema had failed.

Also, this study includes assessments only at initial evaluation and at the conclusion of the intensive phase of treatment. There was no follow-up data available to analyze long-term effects of the intervention or to evaluate the persistent effects of the maintenance phase of treatment.

Future studies may use a randomized control or waitlist control design to confirm the value of mCDT for management of edema in persons with spinal cord injury. They may also include a long-term follow-up measure.

\section{Conclusion}

This study showed that treatment with a mCDT approach can result in meaningful improvements in fluid volume, observational edema characteristics, and pitting quality in edematous lower extremities of individuals with SCI. We found the mCDT approach to be safe, well-tolerated by the patients and feasible in a clinical setting. No adverse responses to the intensive CDT intervention occurred. A 
small number of patients experienced mild, grade 1 pressure areas during the maintenance phase, highlighting the need for monitoring of skin health during this phase. Based on these outcomes, we recommend considering this mCDT approach for management of edema in individuals with SCI, while remaining vigilant about skin inspection.

Author contributions R.H. was responsible for conception of the treatment approach, development of the edema program, treatment of many of the patients and data collection; C.F. contributed content expertize and was responsible for writing the manuscript; W.M.S. performed the statistical analysis and wrote the data analysis section; E.F.F. contributed to the analysis and interpretation of the data, development, and revision of the manuscript, and approval of the final version of the manuscript.

\section{Compliance with ethical standards}

Conflict of interest The authors declare that they have no conflict of interest.

\section{References}

1. Word Health Organization. International Perspectives on Spinal Cord Injury. 2013:250. https://doi.org/10.1007/978-1-4899-1028-8_18.

2. NSCISC. Spinal cord injury facts and figures at a glance. J Spinal Cord Med. 2016;39:493-494.

3. Adriaansen JJE, Ruijs LEM, Van Koppenhagen CF, Van Asbeck F, Snoek G, Van Kuppevelt D, et al. Secondary health conditions and quality of life in persons living with spinal cord injury for at least ten years. J Rehabil Med. 2016;48:853-860.

4. Suehiro K, Morikage N, Murakami M, Yamshita O, Ueda K, Samura M, et al. A study of leg edema in immobile patients. Circ J. 2014;78:1733-1739.

5. Demos Medical Publishing. Spinal Cord Medicine: Principles and Practice.; 2011. https://doi.org/10.1136/jnnp.74.9.1355-a.

6. van der Meer P, Post MWM, van Leeuwen CMC, van Kuppevelt HJM, Smit CAJ, van Asbeck FWA. Impact of health problems secondary to SCI one and five years after first inpatient rehabilitation. Spinal Cord. 2017;55:98-104.

7. Trayes KP, Studdiford JS, Jefferson T, Tully AS, American Family Physician. 2013;88:102-110.

8. Howard SB, Krishnagiri S. The use of manual edema mobilization for the reduction of persistent edema in the upper limb. J Hand Ther. 2001;14:291-301.
9. Bell A, Muller M. Effects of kinesio tape to reduce hand edema in acute stroke. Top Stroke Rehabil. 2013;20:283-288.

10. Dennis M, Sandercock P, Graham C, Forbes J. The Clots in Legs or sTockings after Stroke (CLOTS) 3 trial: a randomised controlled trial to determine whether or not intermittent pneumatic compression reduces the risk of post-stroke deep vein thrombosis and to estimate its cost-effectiveness. Health Technol Assess 2015;19. https://doi.org/10.3310/hta19760.

11. Song S. Swollen Feet and Legs: edema in SCI. Spinal Cord Inj Updat. 2015;24. http://sci.washington.edu/info/newsletters/a rticles/15_spr_edema.asp.

12. Wang CL, Tang FT, Wong MK. A comparison of compression stockings of different pressures with lower leg edema in spinal cord injury or lesions patients. J Formos Med Assoc. 1995;94: S149-55.

13. Casley-Smith JR, Boris M, Weindorf S, Lasinski B. Treatment for lymphedema of the arm - the Casley-Smith method. Cancer. 1998;83:2843-2860.

14. SITZIA J. Volume measurement in lymphoedema treatment: examination of formulae. Eur J Cancer Care. 1995;4:11-16.

15. Dobkin BH. Progressive staging of pilot studies to improve phase III trials for motor interventions. Neurorehabil Neural Repair. 2009;23:197-206.

16. ISL. The diagnosis and treatment of peripheral lymphedema: 2013 consensus document of the international society of lymphology. Lymphology. 2013;46:1-11.

17. Liao SF, Li SH, Huang HY. The efficacy of complex decongestive physiotherapy (CDP) and predictive factors of response to CDP in lower limb lymphedema (LLL) after pelvic cancer treatment. Gynecol Oncol. 2012;125:712-715. https://doi.org/10.1016/j. ygyno.2012.03.017

18. Ezzo J, Manheimer E, McNeely ML, Howell D, Weiss R, Johansson K, et al. Manual lymphatic drainage for lymphedema following breast cancer treatment. Cochrane Database Syst Rev. 2015;5:CD003475

19. Rabe E, Partsch H, Hafner J, Lattimer C, Mosti G, Neumann M, et al. Indications for medical compression stockings in venous and lymphatic disorders: an evidence-based consensus statement. Phlebology. 2017;0:26835551668963

20. Dennis M, Sandercock PA, Reid J, Graham C, Murray G, Venables G, et al.CLOTS Trials Collaboration Effectiveness of thighlength graduated compression stockings to reduce the risk of deep vein thrombosis after stroke (CLOTS trial 1): a multicentre, randomised controlled trial. Lancet. 2009;373:1958-1965.

21. Midttun M, Ahmadzay NF, Henriksen JH. Does comprilan bandage have any influence on peripheral perfusion in patients with oedema? Clin Physiol Funct Imaging. 2010;30:323-327. 